\title{
An Approach to Modification of Water Flow Algorithm for Segmentation and Text Parameters Extraction
}

\author{
Darko Brodić $^{1}$ and Zoran Milivojević ${ }^{2}$ \\ ${ }^{1}$ University of Belgrade, Technical Faculty Bor, V.J. 12, 19210 Bor, Serbia \\ dbrodic@tf.bor.ac.rs \\ ${ }^{2}$ Technical College Niš, Aleksandra Medvedeva 20, 18000 Niš, Serbia \\ zoran.milivojevic@vtsnis.edu.rs
}

\begin{abstract}
This paper proposes an approach to water flow method modification for text segmentation and reference text line detection of sample text at almost any skew angle. Original water flow algorithm assumes hypothetical water flows under only a few specified angles of the document image frame from left to right and vice versa. As a result of water flow algorithm, unwetted image frames are extracted. These areas are of major importance for text line parameters extraction as well as for text segmentation. Water flow method modification means extension values of water flow specified angle and unwetted image frames function enlargement. Modified method is examined and evaluated under different sample text skew angles. Results are encouraged especially due to improving text segmentation which is the most challenging process stage.
\end{abstract}

Keywords: Document image processing, Reference text line, Text line segmentation, Water flow algorithm.

\section{Introduction}

Previous work on text parameter detection can be categorized in few types:

- Histogram analysis,

- Docstrum (k-nearest neighbor clustering),

- $\quad$ Projection profile (Hough transform),

- Fourier transform,

- Cross-correlation,

- Other methods.

In [1] is mentioned previously proposed and accepted technique of reference line extraction based on identifying valleys of horizontal pixel density histogram. Method failed due to multi-skewed text lines.

The Docstrum method [2] is by product of a larger page layout analysis system, which assumed that only text is being processed. The connected components formed by the nearest neighbors clustering are essentially characters only. The method is suitable for finding skew angle. But, it is limited to Roman languages due to poor text line segmentation. 
Another method proposed in [2,3] deal with "simple" multi-skewed text. It uses as a basis simple type of Hough transform for straight lines. But, it's too specific.

The Fourier transform method is a representation in the Fourier domain of the projection profile method in the pixel domain. The results are mathematically identical, but Fourier transform is only different approach to the same text and document properties that projection profile is based upon [2].

The cross-correlation method calculates both horizontal and vertical projection profiles and then compares the shift inter-line cross-correlation to determine the skew rate. Although method can handle complex layout structure documents, applied range is limited to $\left(-10^{\circ}, 10^{\circ}\right)[2]$.

Algorithm proposed by [4] model text line detection as an image segmentation problem by enhancing text line structure using a Gaussian window and adopting the level set method to evolve text line boundaries. Author specified method as robust, but rotating text by an angle of $10^{\circ}$ has an impact on reference line hit rate.

Method of identifying words contour area as a start of detecting baseline point proposed in [5]. The assumptions made on the word elements definition are too specific.

Method [1] hypothetically assumed a flow of water in a particular direction across image frame in a way that it faces obstruction from the characters of the text lines. This method is adopted in $[6,7]$, but referent line hit rate is robust for rotating origin text from $\pm 20^{\circ}$ around $\mathrm{x}$-axis In our paper, "water flow" method $[1,6,7]$ is further adopted, implemented and examined on more complex text examples.

This paper is organized as follows: In section 2 contribution to technological innovation i.e. modification of water flow algorithm is presented. Section 3 includes definition of experiment under investigation. In section 4 experimental results are presented. Results are analyzed, examined, elaborated and discussed as well. In section 5 conclusions are made.

\section{Contribution to Technological Innovation}

Document text image identification procedure consists of three main stages. It is shown in Fig. 1.

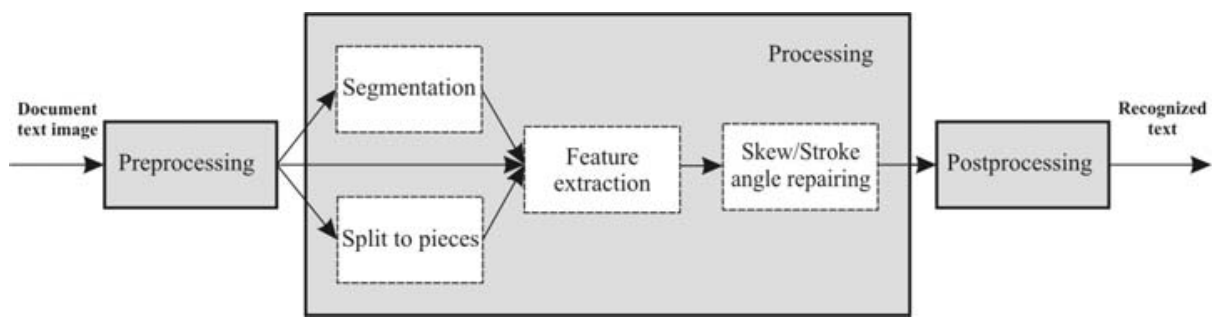

Fig. 1. Document text image identification procedure

In preprocessing stage, algorithm for document text image binarization and normalization is applied. Now, preprocessing text is prepared for segmentation, feature extraction and character recognition. During the processing stage, algorithms for text 
segmentation as well as for skew and reference text line identification are enforced. After that, reference text based on skew and stroke angle, is straightened and repaired. Finally, in postprocessing stage character recognition process is applied.

In this paper, some elements of preprocessing and processing stages are employed. A few assumptions should be made before defining algorithm. We suppose that there is an element of preprocessing. After preprocessing, document text image is prepared for segmentation and feature extraction. So, it represents distinct entity consists of group of words.

Document text image is an input of text grayscale image described by following intensity function:

$$
I(l, k) \in[0, \ldots, 255],
$$

where $l \in[0, \mathrm{~N}-1]$ and $k \in[0, \mathrm{M}-1]$.

After applying intensity segmentation with binarization, intensity function is converted into binary intensity function given by:

$$
I_{b i n}(l, k)=\left\{\begin{array}{lll}
1 & \text { for } & I(l, k) \geq I_{\text {th }} \\
0 & \text { for } & I(l, k)<I_{\text {th }}
\end{array},\right.
$$

where $I_{t h}$ is given by Otsu algorithm [8].

Now, text lines are represented as digitized document image by matrix $\mathbf{X}$ with $\mathrm{M} \times \mathrm{N}$ dimension. Each word in document image consists of black points i.e. pixels. Every point is represented by number of coordinate pairs such as:

$$
X(i, j) \in[0,1],
$$

where $i=1, \ldots, \mathrm{N}, j=1, \ldots, \mathrm{M}$ of the matrix $\mathbf{X}[8,9]$.

Original water flow algorithm assumes hypothetical water flows under only few specified angles of the document image frame from left to right and vice versa [1]. But, previously it needs to define pixel type from situation in Fig. 2.

\begin{tabular}{|c|c|c|}
\hline & $i$ & $i+1$ \\
\hline$j-1$ & $o$ & $o$ \\
\hline$j$ & $x$ & $x$ \\
\hline$j+1$ & $x$ & $o$ \\
\hline
\end{tabular}

(a)

\begin{tabular}{|c|c|c|}
\hline & $i$ & $i+1$ \\
\hline$j-1$ & 0 & 0 \\
\hline$j$ & $x$ & 0 \\
\hline$j+1$ & $x$ & 0 \\
\hline
\end{tabular}

(b)

\begin{tabular}{|c|c|c|}
\hline & $i$ & $i+1$ \\
\hline$j-1$ & 0 & 0 \\
\hline$j$ & $x$ & 0 \\
\hline$j+1$ & $x$ & 0 \\
\hline
\end{tabular}

(c)

Fig. 2. Pixel type determination: (a) Upper boundary pixel, (b) Lower boundary pixel, and (c) Boundary pixel for additional investigation ( $\mathrm{x}$ represents black and o represents white pixel)

Proposed algorithm verifies boundary pixel type in document image. After verification it makes unwetted areas around the words. Due to pixel type, i.e. upper or lower, slope is $\alpha$ or $-\alpha$. Additional investigation is made on pixel without complete location. It can be lower, upper or no boundary pixel. It depends on neighbor area of pixels. Apart from [8] and [9] enlarged window R x S pixels is defined as a basis. For analysis, it is proposed $\mathrm{R}=5$ and $\mathrm{S}=7$ [7]. Position of window is backwards from pixel 
candidate for additional investigation. After additional investigation pixel type is completely located [7]. Throughout previous decision making, unwetted areas algorithm simply draws area under specified angles. As a result words are bounded by unwetted dark stripes. These regions are mark out by lines defined as function:

$$
y=k \cdot x,
$$

where slope $k=\tan (\alpha)$. Lines defined by slope make connection in specific pixel creating unwetted area defined as grey region in Fig. 3.

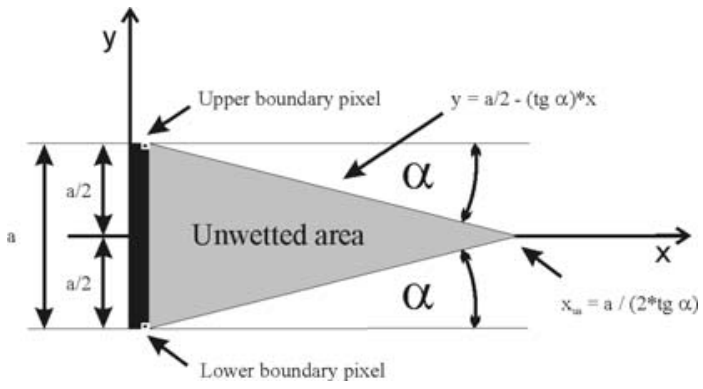

Fig. 3. Unwetted area definition

Basic water flow algorithm is proposed with water flow fixed angles of: $45^{\circ}, 26.6^{\circ}$, $18.4^{\circ}$ and $14^{\circ}$ by masking out original document image [1]. First modification made on water flow algorithm is its extension in formulation of algorithm. Still, making straight lines from boundary pixel type and connecting each others in specified point makes unweted region as well. Hence, modified water flow algorithm is free to choose different $\alpha$ from $0^{\circ}$ to $90^{\circ}$. Unfortunately, whole range of $\alpha$ isn't applicable due to impossibility of joining words to form text line regions.

Further improvement is made on defining water flow function from (4). From mathematical calculus it is known that (4) is special case of more universal function:

$$
y=k \cdot x^{n},
$$

where (4) and (5) are equal for $n=1$.

Using different $n$ values from (5) rebuild different shape of unwetted areas around the words. The main achieving of unwetted area is to be exploited for text segmentation. The problem lies in broken words in text lines. Algorithm should join those words by unwetted areas. Unwetted areas can lengthen by using smaller angle $\alpha$ or different water flow function. Hence, another modification of algorithm function means more slow down of slope forming unwetted area. Expanded unwetted area is given in Fig. 4.

Water flow function is changed from (4) to (5) with power value $0<n<1$.

Basic and expanded water flow function zeroes are marked as $x_{u a}$ and $x_{\text {eua }}$, respectively. Their difference $x_{\text {diff }}=x_{\text {eua }}-x_{\text {иа }}$ is given by:

$$
x_{d i f f}=\frac{a}{(2 \cdot \tan \alpha)^{\frac{1}{n-1}}} .
$$




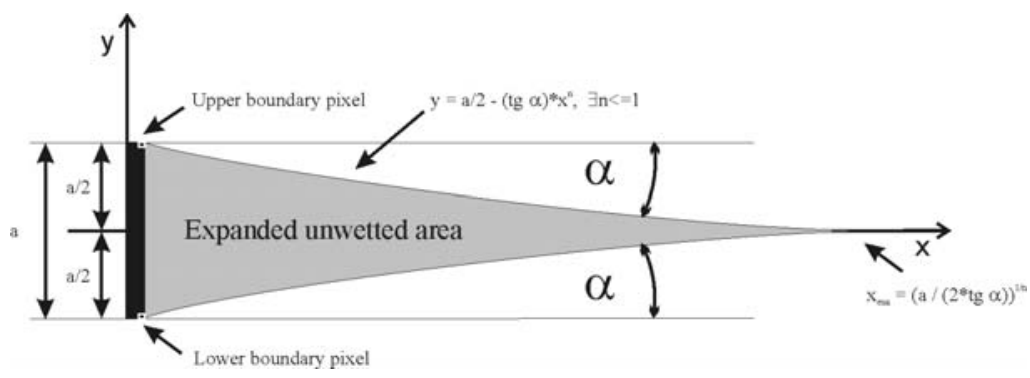

Fig. 4. Expanded unwetted area definition

For parameter $n<1, x_{\text {diff }}$ value is greater than 0 . Thus, unwetted area is expanded. The skew angle detection is based on information obtained from presented algorithm. Defining reference text line means calculating specific average position of every column of document image. It is average position of only black pixels in every column of document image. Relation for calculating reference text line is given by [1]:

$$
x_{i}={\frac{\sum_{j=1}^{L} y_{j}}{L}}_{i=1, \ldots, K},
$$

where $x_{i}$ is calculated point represent reference text line point, $i$ is specified column, $y_{j}$ is position of black pixel in column $j$ and $L$ is sum of black pixel in specified column $j$ of the document image.

\section{Experiment}

For the experiment, sample text rotated from $-45^{\circ}$ to $+45^{\circ}$ by step of $5^{\circ}$ around $x$-axis is used. Sample text is given in Fig. 5. Sample text reference line is represented by:

$$
y=a \cdot x+b .
$$

After applying algorithm, calculated document image from (7), with only one black pixel per column, is obtained. That black pixel per column defines calculated reference text line and text line skewness. Calculated reference text line forms continuous or discontinuous line partly or completely representing reference text line.

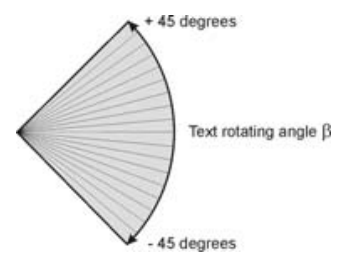

Fig. 5. Sample text rotating from $-45^{\circ}$ to $+45^{\circ}$ for the algorithm robustness investigation 
To achieve continuous linear aspect of reference text line from point's collection, least square method is used. To find a first degree polynomial function approximation given by:

$$
y=a^{\prime} \cdot x+b^{\prime},
$$

number of data points is used as $n d p$ and the slope $a^{\prime}$, and the y-intercept $b^{\prime}$ are calculated as [10]:

$$
a^{\prime}=\frac{\left(\sum y\right) \cdot\left(\sum x \cdot y\right)-n d p \cdot\left(\sum x \cdot y\right)}{\left(\sum x\right)^{2}-n d p \cdot\left(\sum x^{2}\right)},
$$

and

$$
b^{\prime}=\frac{\left(\sum x\right) \cdot\left(\sum x \cdot y\right)-\left(\sum y\right) \cdot\left(\sum x^{2}\right)}{\left(\sum x\right)^{2}-n d p \cdot\left(\sum x^{2}\right)} .
$$

Further, referent line hit rate abbreviated by $R L H R$ is defined by:

$$
R L H R=1-\frac{\beta_{r e f}-\beta_{e s t}}{\beta_{r e f}},
$$

where $\beta_{\text {ref }}$ is arctangent of $a$ (origin) from (8) and $\beta_{\text {est }}$ is arctangent of $a^{\prime}$ (calculated i.e. estimated) from (9). $R M S$ values are calculated by [10]:

$$
R M S=\sqrt{\frac{1}{R} \sum_{i=1}^{R}\left(x_{r e f}-x_{e s t}\right)^{2}},
$$

where $R$ is number of examined text rotating angles range from $\pm 60^{\circ}, x_{\text {ref }}$ is $R L H R$ for $\beta_{e s t}$ equal to $\beta_{\text {ref }}$ i.e. due to normalization equal to 1 , and $x_{e s t}$ is $R L H R$.

\section{Results and Discussion}

Benefit from extended algorithm is perceived in text segmentation process. Split up words in text line is presumably joined by unwetted areas from water flow function. Two word connections made by basic and extended algorithm are given in Fig. 6.

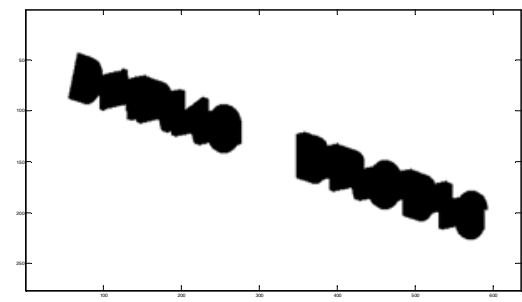

(a)

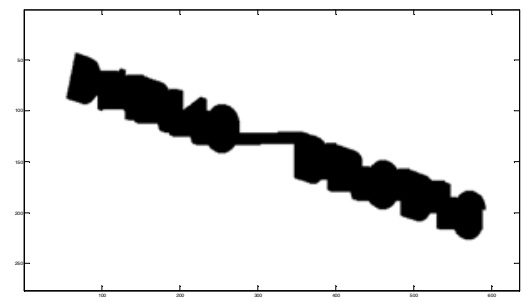

(b)

Fig. 6. Document text image with unwetted areas: (a) basic algorithm, and (b) extended algorithm (power $n=1 / 2$ from (5)) 
Sample text $R L H R$ for basic and extended algorithm is given in Fig. 7.

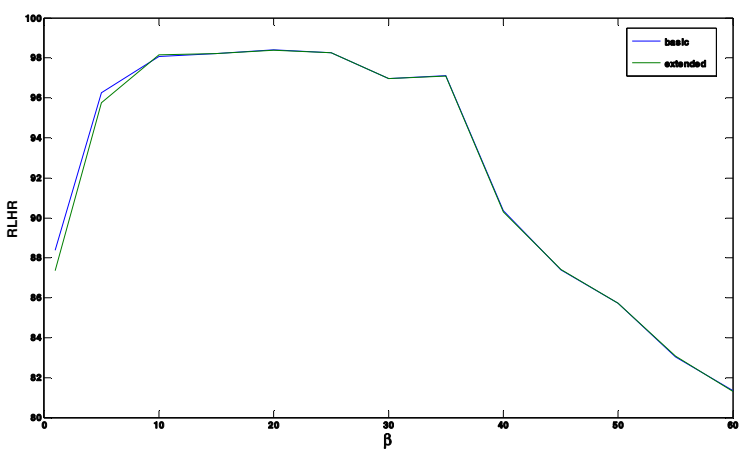

Fig. 7. Sample text $R H L R$ ( $\alpha$ from $10^{\circ}$ to $30^{\circ}$ by step $5^{\circ}, \beta$ from $5^{\circ}$ to $60^{\circ}$ by step $5^{\circ}$ ): basic vs. extended algorithm $(n=1 / 2)$

$R L H R$ for basic and extended algorithm are almost identical as can be seen from Fig. 7. For sample text rotating up to $40^{\circ} R L H R$ is at least $90 \%$ or better. It proved robustness of both algorithms. RLHR isn't reduced due to better segmentation by extended algorithm. Sample text $R M S$ for basic and extended algorithm is given in Fig. 8.

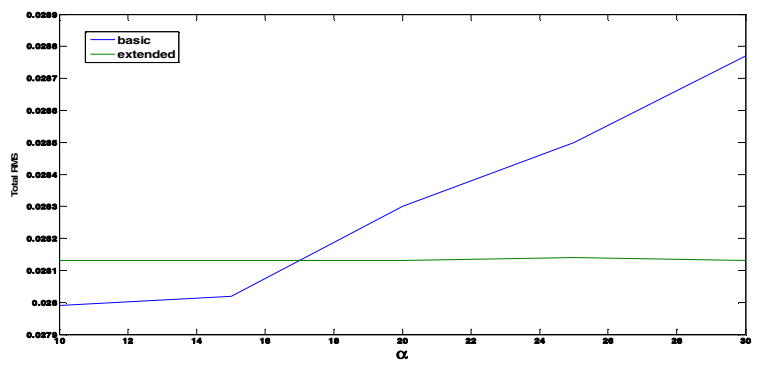

Fig. 8. Sample text $R M S$ ( $\alpha$ from $10^{\circ}$ to $30^{\circ}$ by step $5^{\circ}, \beta$ from $5^{\circ}$ to $60^{\circ}$ by step $5^{\circ}$ ): basic algorithm vs. extended algorithm $(n=1 / 2)$

$R M S$ made by basic algorithm for $\alpha$ from $10^{\circ}$ to $30^{\circ}$ is growing on. Unlike, $R M S$ made by extended algorithm is steady. Due to $R M S$ condition, magnitude of varying quantities is similar for the whole investigated region of the unwetted angles in extended algorithm.

\section{Conclusion}

In this paper an approach to modified water flow algorithm for text segmentation and reference text line extraction is presented. Water flow algorithm assumes hypothetical 
water flows under few specified angles of the image frame from left to right and vice versa. As a result of algorithm, unwetted image regions defined by function $y=k x$ are restored. Modified water flow algorithm made water to flow under whole range of different angles. Same way, it exchanges function defining unwetted image regions to $y=k x^{n}$. Those unwetted regions are corner stone needed for reference text line calculation. Using least square method on calculated reference text line, reference text line is defined and extracted. Modified water flow function proved better text segmentation preferences. Robustness of algorithm is examined and validated using sample text rotating in region of $\pm 60^{\circ}$ around x-axis. Results confirmed good $R L H R$ values for text rotating in region of $\pm 45^{\circ}$. Further improvements of algorithm should be made on better $R L H R$ in full region of $\pm 90^{\circ}$. One way is algorithm throw out to strictly follow reference text line turn over.

\section{References}

1. Basu, S., Chaudhuri, C., Kundu, M., Nasipuri, M., Basu, D.K.: Text Line Extraction from Multi-Skewed Handwritten Documents. Pattern Recognition 40(6), 1825-1829 (2006)

2. Amin, A., Wu, S.: Robust Skew Detection in mixed Text/Graphics Documents. In: Proceedings of 8th International Conference on Document Analysis and Recognition (ICDAR 2005), Seoul, Korea, pp. 247-251 (2005)

3. Louloudis, G., Gatos, B., Pratikakis, I., Halatsis, C.: Text Line Detection in Handwritten Documents. Pattern Recognition 41(12), 3758-3772 (2008)

4. Li, Y., Zheng, Y., Doermann, D., Jaeger, S.: A New Algorithm for Detecting Text Line in Handwritten Documents. In: Proceedings of 18th International Conference on Pattern Recognition, Hong Kong, China, vol. 2, pp. 1030-1033 (2006)

5. Wang, J., Mazlor, K., Leung, H., Hui, S.C.: Cursive Word Reference Line Detection. Pattern Recognition 30(3), 503-511 (1997)

6. Brodić, D., Milivojević, Z.: Reference Text Line Identification Based on Water Flow Algorithm. In: Proceedings of ICEST 2009, SP-2 Sect, Veliko Tarnovo, Bulgaria (2009)

7. Brodić, D., Milivojević, Z.: Modified Water Flow Method for Reference Text Line Detection. In: Proceedings of ICCS 2009, Sofia, Bulgaria (2009)

8. Gonzalez, R.C., Woods, R.E.: Digital Image Procesing, 2nd edn., pp. 67-70. Prentice-Hall, New Jersey (2002)

9. Sonka, M., Hlavac, V., Boyle, R.: Image Processing, Analysis and Machine Vision, pp. 174-177. Thomson, Toronto (2008)

10. Bolstad, W.M.: Introduction to Bayesian Statistics, pp. 40-44, 235-240. John Wiley \& Sons, New Jersey (2004) 